\title{
1 Encapsulation of plant-derived bioactive ingredients through \\ 2 electrospraying for nutraceuticals and functional foods applications
}

3 Laura G. Gómez-Mascaraque ${ }^{1}$, Amparo Lopez-Rubio ${ }^{2 *}$

$4 \quad{ }^{1}$ Teagasc Food Research Centre, Moorepark, Fermoy, Co. Cork, Ireland

5 email: laura.mascaraque@teagasc.ie; Tel.: +353761112241

$6 \quad{ }^{2}$ Food Preservation and Food Quality Department, IATA-CSIC, Avda. Agustin

7 Escardino 7, 46980 Paterna, Valencia, Spain

8 email: amparo.lopez@iata.csic.es; Tel.: +34 963900022

9

10 ABSTRACT

11 The electrospraying technique, which consists of electrohydrodynamic atomization of polymeric fluids, can be used to generate dry nano- and microparticles by subjecting a polymer solution, suspension or melt to a high voltage electric field. This potential can be exploited for developing nano- and microencapsulation structures under mild temperature conditions and, thus, constitutes a promising alternative to conventional microencapsulation techniques for sensitive ingredients, like most plant-derived bioactive compounds, especially for their application in the food sector. Given the importance of plants as one of the major sources of dietary bioactive compounds, much research attention has been recently paid to the encapsulation of phytochemicals through novel techniques such as electrospraying, aiming to provide new tools for the development of innovative functional food products and nutraceuticals. In this review, the latest advances in the application of electrospraying for nano- and microencapsulation of phytochemicals are discussed, with a focus on their potential use in the food sector. 
27 Phytochemicals have been widely recognised for their contribution to the health-promoting effects of plant-based diets [1]. Consequently, the interest in exploiting them for food products enrichment or fortification has been boosted in the last years in response to the increased demand for functional foods and beverages [2]. However, most plant-derived bioactive ingredients are sensitive to different environmental stresses, such as high temperatures, light, oxygen, certain $\mathrm{pH}$ conditions, etc., so they can lose their expected beneficial bioactivity during food processing, storage and/or consumption [3]. Moreover, addition of bioactive ingredients to food products in which they are not normally found involves other challenges, which include possible changes in the organoleptic properties of the final food products, incompatibilities between the added ingredient and the food matrix, or low bioavailability of the active compounds to be incorporated [4-6]. In this context, nano- and microencapsulation are promising approaches to overcome the aforementioned limitations for developing new enriched functional foods and nutraceuticals. These technologies consist of coating or embedding the ingredient of interest within a nano- or micron-sized protective matrix $[7,8]$, which works as a physical barrier between the bioactive compound and the food matrix or the environment [9]. Moreover, interactions between the ingredient and the protective matrix can take place, further contributing to stabilizing the bioactive compounds.

A number of nano- and microencapsulation methods have been already developed, many of which can be used for food applications [10]. However, most of them face a series of disadvantages, such as the use of toxic solvents or reagents, the need of high temperatures for processing, which may damage sensitive compounds, the low microencapsulation efficiencies achieved, the occurrence of particle agglomeration or the high costs or even non-scalability of the process [11-14]. Moreover, most of them need further processing after production, 
especially drying to increase shelf-stability of the formulated ingredients [15] so, in general, they are ultimately used in combination with one of the drying encapsulation techniques [16, 17]. In fact, drying technologies, mainly spray-drying and freeze-drying, are the most widely employed microencapsulation technologies in the food industry [12]. However, due to the high temperatures required for spray-drying, this technique is unsuitable for many phytochemicals which are thermosensitive [18]. On the other hand, freeze-drying is considerably more expensive and time consuming [12], and yields highly porous materials with insufficient barrier properties [14].

In this context, electrospraying is a promising alternative which has been very recently proposed for food applications, and can be carried out under mild conditions [19]. This technology consists of applying a high voltage electric field to a polymeric fluid in order to spray and dry it in a single step, generating nano- and/or microparticles suitable for encapsulation. Although it was initially explored for drug delivery purposes [20], its applications were later on extended to other fields including the food industry and, in particular, the development of new functional foods and nutraceuticals $[3,18,21]$, were phytochemicals play a relevant role.

Electrospraying is a very versatile technology which offers a number of advantages over other microencapsulation methods. It has the benefits of a drying technique, since it allows obtaining dry nano- or microparticles in a one-step process [22] and this can be achieved without applying high temperatures nor freezing [23]. Moreover, its operation is quite easy and cost-effective, and it generally achieves uniform dispersion of the ingredients within the wall materials with high loading capacities and minimal compound losses [24]. The particle size of the obtained capsules is also generally smaller than that obtained with conventional 
atomizers, and with narrower size distributions, and particle aggregation is, in principle, prevented by electrical repulsion between charged droplets [25] although, in practice, aggregation is commonly observed mainly due to insufficient solvent evaporation, especially when using aqueous solutions.

This review briefly describes the fundamentals of the electrospraying technique and its application for the production of edible encapsulation structures of interest for the food industry. Additionally, the state of the art on the encapsulation of plant-derived bioactive compounds through this technology and their potential use as functional ingredients or nutraceuticals is also reviewed.

\section{Description and basics of the electrospraying technique}

Electrospraying, also referred to as electrohydrodynamic atomization, is a drying technique based on electrically processing charged fluids to generate dry, fine powders in the form of nano- and/or microparticles. This is achieved by subjecting a polymer solution, dispersion or melt to a high voltage electric field [26]. Generally, the polymeric fluid is pumped through a needle or a capillary nozzle oppositely placed to a grounded collector at a certain distance, as illustrated in Figure 1. Upon application of an electric field between nozzle and collector, the fluid drop, which is flowing out of the nozzle, is subjected to a higher electrostatic potential than its surroundings [27], developing a pointed meniscus known as Taylor cone [28]. When the electric forces (namely Coulomb forces) generated in the fluid dominate over its surface tension and cohesion forces, a charged jet is ejected towards the collector [29]. During the flight, the solvent in the jet is evaporated, thus rendering a dry material which is finally obtained in the collector [30]. 


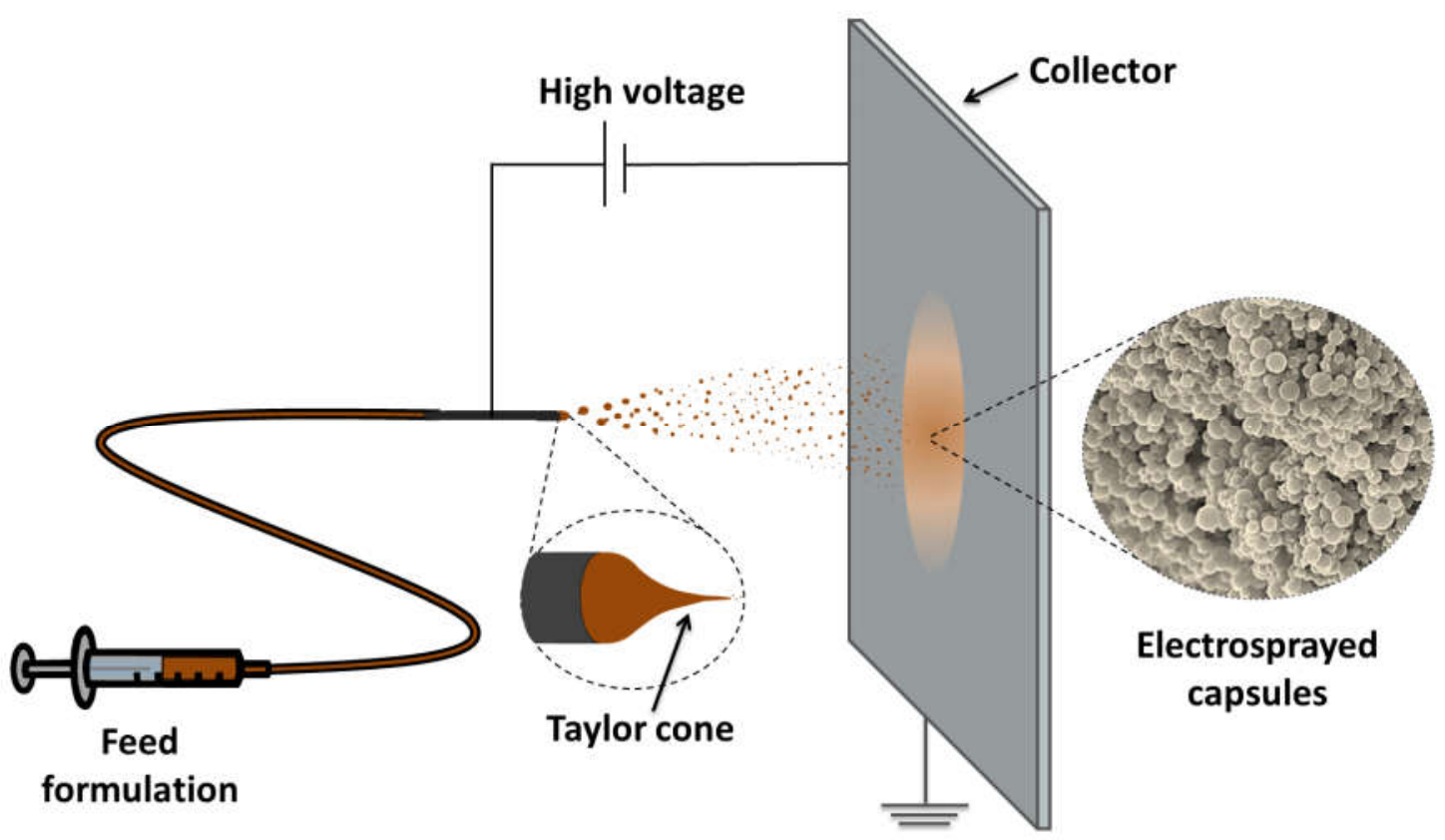

101

102

103

104

105

106

107

108

109

111

112

113

114

115

116

117

Figure 1. Scheme of a basic electrospraying set-up.

Depending on the solution properties of the fluid and the process parameters used, a range of different morphologies can be obtained for the dried materials produced through electrohydrodynamic processing [24]. Electrospraying is the particular case in which fine particles are obtained, and it takes place when the intermolecular cohesion of the polymeric fluid is low enough so that the electric forces can break the jet into fine droplets, giving rise to nano- or microparticles upon drying [31]. Conversely, if the molecular cohesion is sufficiently high to prevent fragmentation of the jet, it elongates yielding ultrathin fibres upon solvent evaporation [32]. In this latter case, the process is referred to as electrospinning, and is generally used to produce non-woven fibre matts, films or coatings [33, 34]. Hence, electrospraying and electrospinning are two variant modes of the electrohydrodynamic processing technique which differ in the morphology of the obtained nano- and microstructures. In general, for nutraceuticals and functional food applications, powder-like formulated ingredients are preferred over continuous fibrillar materials, since they are easier to handle and to disperse within food matrices [3]. As a result, the solution properties of the 
118 feed formulations and the processing parameters need to be carefully optimized in order to

119 result in adequate conditions for electrospraying.

121 It is also worth mentioning that similar setups and principles as those used for electrospraying are also exploited for the electrostatic extrusion encapsulation technique, sometimes known as electrospraying-aided or electrospray-assisted extrusion [35], in which an external electric field is similarly used to break up fluid droplets to reduce their size before gelling them. Although some authors consider it as a variant of the electrospraying technique [29], it differs from conventional electrospraying in that it does not produce dry capsules. Instead, electrostatic extrusion is a wet microencapsulation technique which yields micron-sized hydrogels and, thus, it will not be considered in this review.

Due to the narrow limit between the electrospraying and electrospinning modes of hydrodynamic processing technologies which, however, yield materials with very different applications, some authors have tried to generalize the ideal properties and parameters ranges for operating in one mode or another. As an example, Doshi and Reneker (1995) suggested that, in order to achieve electrospinning conditions, the viscosity of the fluid should be in the range of $800-4000 \mathrm{cP}$, as lower values were considered too diluted to achieve enough chain entanglements [36]. However, nanofibres were later on produced from solutions with lower viscosities [3]. Similarly, while in general an increase in the applied voltage usually results in a reduction of the particles diameter, exceptions have also been observed [32]. Jaworek and Sobczyk (2008) described the balance of forces (electrodynamic, inertial, drag and gravitational) involved in the electrospraying process but, given their complexity, solutions for

141 those general equations have not been provided yet [25]. As a consequence, and given the 142 great variety of polymer systems available, with different molecular structures, physical- 
chemical properties and entanglement behaviour in a selected solvent, the optimum ranges need to be determined for each feed formulation [32].

It is widely accepted that the characteristics of the feed formulations which affect the electrospraying process to a greater extent are their rheological properties, electrical conductivity and surface tension, which are determined by the composition and concentration of the polymeric fluid. Gómez-Mascaraque, et al. (2016) reported that, using these three parameters, the morphology (capsules vs. fibers) obtained upon electrohydrodynamic processing of chitosan solutions with different concentrations and molecular weights could be correctly predicted for almost $85 \%$ of the formulations they tested [37]. In general, fibres can be obtained by increasing the viscosity over a critical value [38], while lower viscosities favour the break-up of the jet into droplets resulting in electrosprayed capsules [32]. On the other hand, increasing electrical conductivities facilitate the elongation of the jet to form fibres (within the limits of jet stability), while increasing surface tension tends to favour jet break up [38]. Other relevant factors include the volatility of the solvent, which determines the rate of evaporation and, thus, the morphology and potential aggregation of the final materials.

Regarding the process parameters, the main factors having an impact on the electrospraying process are the magnitude of the applied electric field, the feed flow-rate, the tip-to-collector distance and the diameter of the nozzle. An increase in the applied voltage or the tip-tocollector distance generally results in a reduction of the particles diameter, while an increase in the diameter of the nozzle or the flow rate usually yields bigger particles. Nevertheless, as previously mentioned, the optimal ranges need to be determined for each formulation. Environmental conditions such as the temperature or the relative humidity also need to be taken into account, as they affect the rate of solvent evaporation [25, 32]. 


\section{Electrospraying of edible biopolymers for food applications}

171 Although electrospraying is a relatively mature research field, its application to develop food products still involves a series of challenges. The food sector is very restrictive and, as only food-grade solvents and materials can be used to avoid toxicity problems, the use of water as solvent is almost imperative [39]. However, water has a high surface tension, conductivity and boiling point, which are not the most adequate characteristics for electrospraying [40]. Therefore, the composition of the polymeric fluids to be processed needs to be thoroughly optimized to achieve electrosprayable formulations [23]. One strategy to improve the properties of aqueous formulations is the addition of compounds with surface active properties to lower their surface tension. This approach proved to be successful to obtain electrosprayed capsules suitable for food applications [40, 41].

Evidently, the encapsulation matrices also need to be suitable for human consumption if they are expected to be used for food applications, so only edible biopolymers (like proteins and polysaccharides) can be considered for this purpose. However, some biopolymers are also challenging to electrospray due to their particular chemical and structural features. A number of them have a poly-ionic nature which results in too conductive solutions, their molecular weight is often too high or too low to achieve the adequate extent of chain entanglements for electrospraying, and many of them also have low chain flexibilities [23]. Several strategies have been proposed to modify the conformation of the biopolymeric chains and/or the extent of intermolecular interactions among them, such as protein denaturation [41] or $\mathrm{pH}$ modulation [3]. 
194 food applications have been already successfully electrosprayed in the last decades. Figure 2

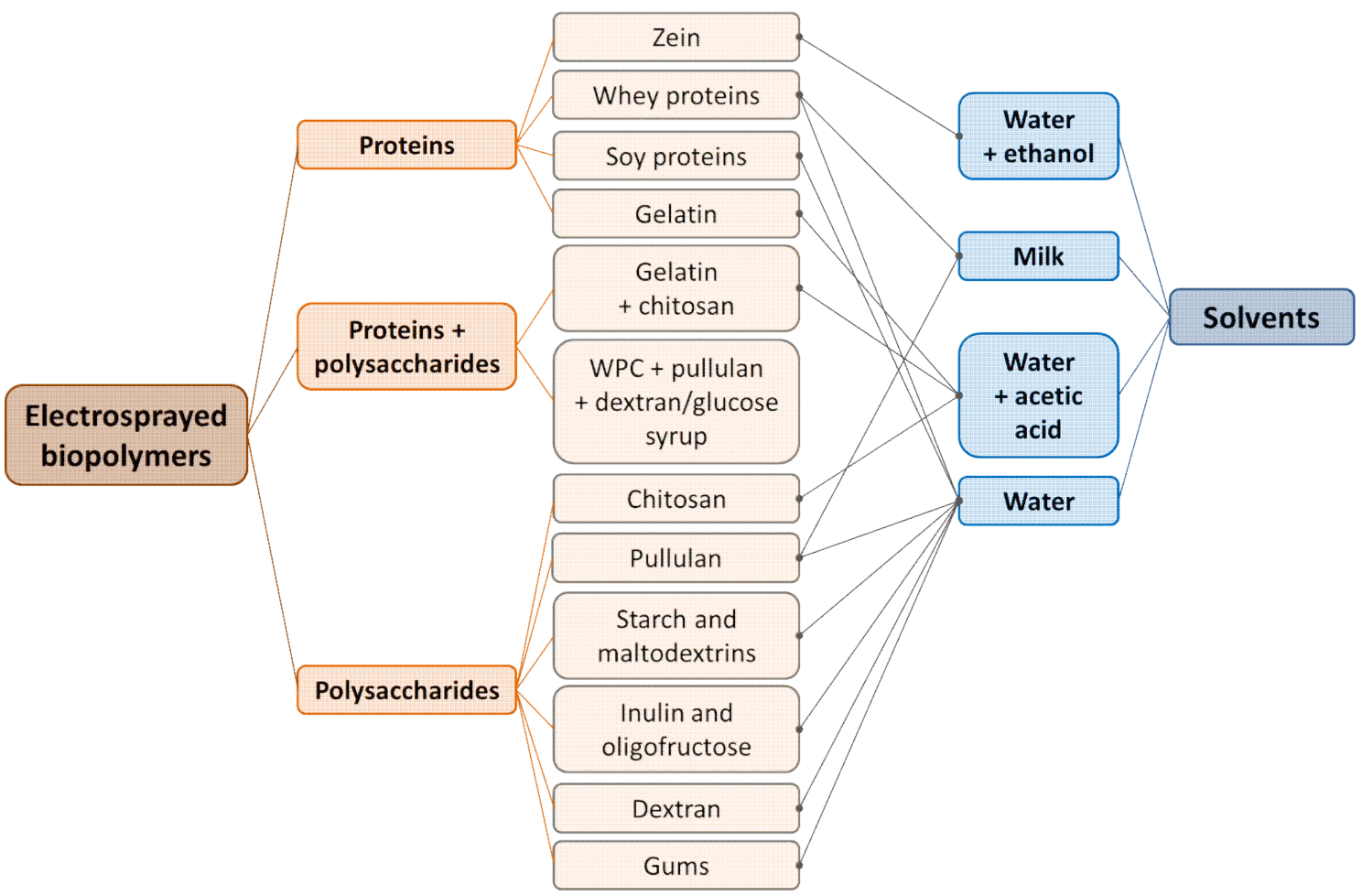

Figure 2. Edible biopolymers and permitted solvents used to produce electrosprayed encapsulation structures with potential application for functional foods and nutraceuticals

\subsection{Electrospraying of protein-based formulations}

Proteins are attractive wall materials for encapsulation purposes in the food industry due to

their intrinsic nutritional value [42]. Moreover, for their processing through electrospraying, they are especially interesting, since their amphiphilic nature [43] helps lowering the surface tension of aqueous formulations [44], apart from aiding in the stabilization of oil-in-water emulsions for the encapsulation of hydrophobic compounds [45]. A number of proteins have been explored for the encapsulation of food ingredients through electrospraying, including zein, whey proteins, soy proteins or gelatin. 
210 Zein, the main protein found in corn, was the first biopolymer exploited to produce edible

211 electrosprayed capsules [46], due to its good solubility in ethanol. Given that this solvent has a

212 lower surface tension, electrical conductivity and boiling point than water, ethanolic solutions

213 are usually easier to process by electrohydrodynamic techniques, and its controlled use for

214 food applications is permitted. Electrosprayed zein has been utilized as encapsulation matrix

215 for $\omega-3$ fatty acids[46] , carotenoids [47] and dietary polyphenols [48-50]. According to Gomez-

216 Estaca, et al. (2012), electrosprayed zein capsules with different morphologies could be

217 produced from a wide range of protein concentrations, i.e. 2.5 wt. $\%$ - 15 wt.\%, obtaining

218 bigger particle sizes for higher polymer concentrations and flow rates [51].

219 Electrosprayed zein particles have been produced not only using the regular uniaxial configuration [48, 49, 51], but also coaxial setups [50, 52]. Coaxial (and multiaxial) electrospraying offers the possibility of producing core-shell (or multi-layered) nano- or microcapsules $[53,54]$ by processing two (or more) different fluids which are pumped through concentric capillaries. Gómez-Mascaraque, et al. (2018) made use of a coaxial configuration to provide zein-based cores containing bioactive ingredients with an additional protective layer of zein or gelatin [50]. Liu, et al. (2018), on the other hand, proposed a coaxial approach to avoid the clogging of the nozzle which occurs due to the fast drying of zein solutions. Their strategy consisted of pumping just ethanol through the outer orifice of the nozzle, which also resulted in more homogeneous electrosprayed zein particles than those obtained by uniaxial electrospraying [52].

Together with zein, whey proteins are the most commonly used protein matrices for encapsulation through electrospraying, and they can be processed using water as solvent. 
López-Rubio and Lagaron (2012) first proposed the use of electrosprayed whey protein

234 concentrate (WPC) particles as encapsulation vehicles and selected $\beta$-carotene as a model

235 functional ingredient [39]. Soon after, other works exploited the potential of this system to encapsulate other bioactive compounds, including vitamins [21], other carotenoids [55] and $\omega$ 3 fatty acids [56]. Moreover, WPC has demonstrated a generally better performance as an electrosprayed encapsulation vehicle (i.e. greater microencapsulation efficiency and or protective ability) when compared to other electrosprayed biopolymers $[21,55,57]$.

Due to its good electrosprayability and performance as wall material, WPC has been the protein of choice to explore different encapsulation strategies through electrospraying. For instance, López-Rubio, et al. (2012) successfully obtained electrosprayed WPC capsules from dispersions of the protein in milk instead of water [57]. These encapsulation structures have demonstrated to be especially interesting for the protection of probiotic microorganisms. Pérez-Masiá, et al. (2015) proposed the use of the coaxial configuration to encapsulate a hydrophobic carotenoid (pumped through the inner orifice of the nozzle) within a WPC matrix (pumped through the outer orifice) [55]. Emulsion-electrospraying, an alternative approach to disperse lipophilic ingredients within electrosprayed capsules obtained from aqueous protein formulations, has also been successfully used to encapsulate carotenoids and polyunsaturated fatty acids (PUFAs) $[47,56]$ within WPC microcapsules. Recently, a novel dual encapsulation strategy based on the entrapment of lipophilic compounds within liposomes and their further incorporation within electrosprayed proteins was developed using WPC as the selected wall material $[58,59]$.

Gelatin, a protein obtained from the partial hydrolysis of collagen, has been also widely exploited as a wall material for electrospraying. Its ability to form thermo-reversible hydrogels 
makes it especially attractive for encapsulation purposes, since it allows its processing from aqueous solutions while preventing the dissolution of the obtained capsules below their gel-sol transition temperature [60]. However, in order to electrospray gelatin at room temperature, its solutions need to be acidified to prevent gelation $[61,62]$. This somewhat limits the range of bioactive ingredients which can be encapsulated using this matrix through electrospraying, as those sensitive to low pHs may lose their activity $[56,63]$. Nevertheless, it is an ideal candidate for the encapsulation of compounds which are more stable under acidic conditions, such as some dietary polyphenols $[3,50,64]$.

Soy proteins are plant-derived alternative wall materials which have also been successfully electrosprayed, although not in their native form. In order to obtain electrosprayed capsules from aqueous dispersions of a soy protein isolate (SPI), Pérez-Masiá, et al. (2014) proposed a strategy consisting of thermally denaturing the proteins and adding non-ionic surfactants to the formulations [41]. This approach was later on exploited for the encapsulation of PUFAs within electrosprayed SPI capsules through emulsion-electrospraying [56].

\subsection{Electrospraying of carbohydrate-based formulations}

Although the surface active properties of carbohydrates are, in general, inferior than those of proteins, polysaccharides generally exhibit a greater structural stability [65]. Moreover, a number of them are resistant to enzymatic cleavage, not being digested in the upper gastrointestinal tract [66]. These characteristics, together with their abundance and relatively low price [67], make carbohydrates interesting encapsulation matrices for electrospraying too.

A number of edible polysaccharides have been successfully electrosprayed from aqueous solutions to date, including starch and maltodextrins, inulin and oligofructose, dextran or 
pullulan $[40,41]$. In order to improve the electrosprayability of some of them, their solution properties (mainly viscosity and surface tension) had to be modified by adding surfactants or gums. In the case of pullulan, its electrospraying has also been performed using skimmed milk as solvent [39]. Some of these electrosprayed systems have proved to be very useful to encapsulate functional ingredients such as vitamins [21] or carotenoids [18].

Due to the poor emulsifying properties of most polysaccharides, coaxial electrospraying may

be a suitable alternative to emulsion-electrospraying for the encapsulation of lipophilic ingredients within electrosprayed carbohydrates. This was the case for the encapsulation of lycopene using electrosprayed dextran, where higher microencapsulation efficiencies were achieved using the coaxial configuration [55].

Gums, which are complex mixtures of polysaccharides containing small fractions of polypeptides are, in general, difficult to electrospray [68], due to their complex molecular structures. Nonetheless, some of them have been successfully used to generate encapsulation structures through this technique too. That is the case of Alyssum homolocarpum seed gum [69] or gum arabic [70], whose electrosprayabilities were enhanced by adding non-ionic surfactants and modulating their solution properties by strategies such as autoclaving the biopolymer solutions or using different methods to prepare the emulsions.

301

Other polysaccharides require the use of water/acetic acid mixtures as solvents to be electrosprayed. This is the case of chitosan [71], which needs their amino groups to be protonated in order to dissolve it in aqueous media [72]. Therefore, similarly to gelatin, electrosprayed chitosan is not a suitable matrix for all functional ingredients [55], although it is perfectly adequate for those with improved stability at low pHs [37]. Ternary water/acetic 
acid/ethanol mixtures can also be used to electrospray chitosan [73]. Due to the usually high molecular weight of this polysaccharide, and consequently its high viscosity in solution, the concentrations of this polymer which can be usually processed by electrospraying are quite low $[74,75]$. In fact, the range of molecular weight-concentration combinations which yield capsules free of nanofibrils is very limited [37].

\subsection{Electrospraying of protein/carbohydrate mixtures}

The use of biopolymer mixtures and, especially, blends of polysaccharides and proteins, is also of great interest for microencapsulation purposes, since it allows modulating the properties of the obtained materials by modifying their composition [60]. Moreover, the possibility of tuning the solution properties of the feed formulations by altering the ratio between biopolymers in the blends is particularly attractive for the electrospraying technique. However, limited work has been reported on electrosprayed protein/carbohydrate blends to date.

Atay, et al. (2017) explored gelatin/chitosan blends as potential microencapsulation vehicles for polyphenol-rich plant extracts, since both biopolymers can be electrosprayed from solutions in aqueous acetic acid. They found that the release profile of the ingredients from the developed capsules could be modulated by altering the composition of the blends. Specifically, increasing the chitosan content of the formulations limited the release of the polyphenols [76]. García-Moreno, et al. (2018) also studied the electrosprayability of polysaccharides/protein blends, namely WPC/pullulan/dextran and WPC/pullulan/glucose syrup mixtures, although the content of the proteins in the formulations was only about 5 wt.\%. Different compositions were tested in order to obtain neat particles free of residual fibrils [77]. 


\section{Applications for the encapsulation of plant-derived ingredients}

333 Due to the attributed health-promoting benefits and consequent interest in phytochemicals as functional ingredients [78], a number of them have been proposed as potential candidates for the enrichment or fortification of food products [79]. Their encapsulation, aimed at enhancing their bioefficiency [80], has been attempted through different methods [81], among which electrospraying has gained increasing attention in the last decades. Figure 3 summarizes the plant-derived bioactive ingredients which have been encapsulated through this electrohydrodynamic technique to date.

- Lycopene

- $\beta$-Carotene

\section{Plant-derived ingredients encapsulated through electrospraying}

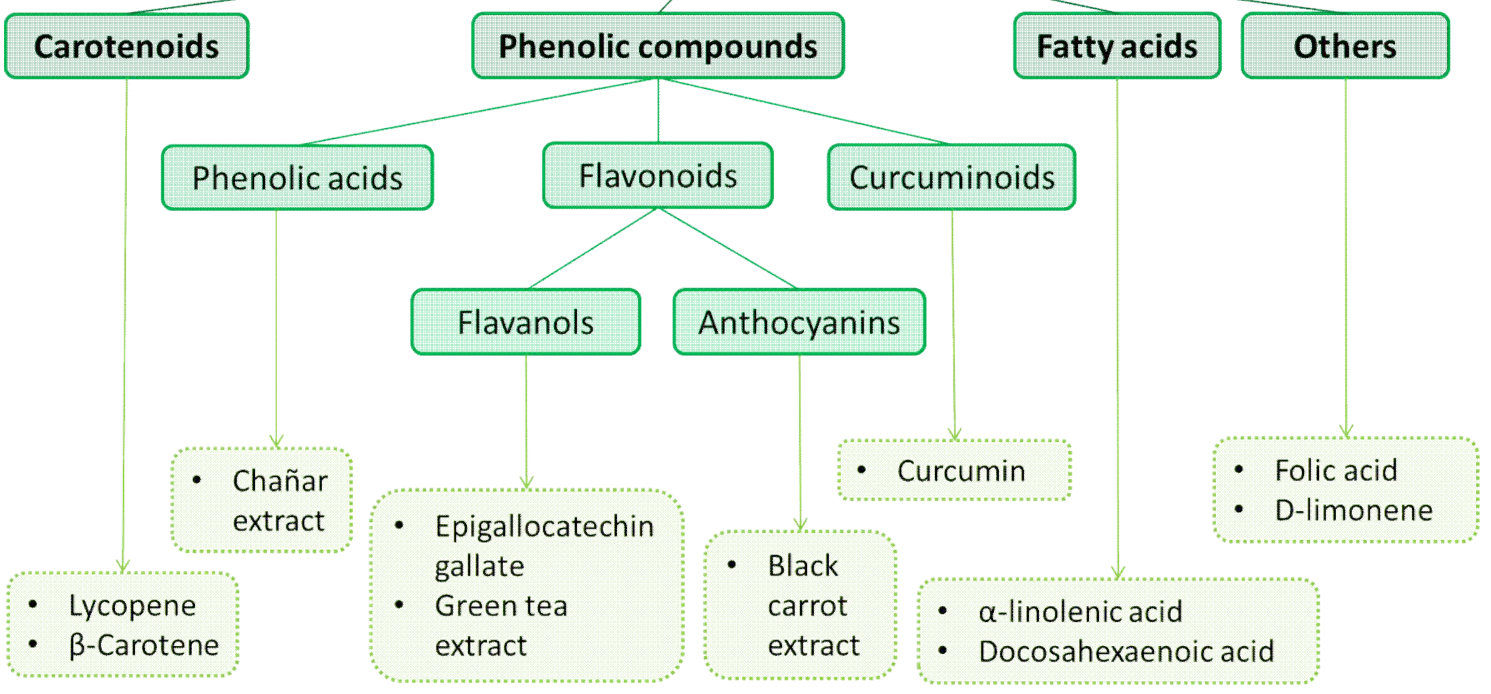

Figure 3. Summary of bioactive phytochemicals which have been encapsulated through electrospraying

\subsection{Encapsulation of phenolic compounds}

Phenolic compounds are bioactive phytochemicals containing, at least, one aromatic ring with various hydroxyl groups, and they exhibit antioxidant and anti-inflammatory activities. High intakes of these compounds have been related to a reduced risk of cancer and cardiovascular diseases and may even slow down some aging processes by protecting against cellular damage 
$350[1,82]$. However, the stability of these compounds has been reported to be generally lower 351 compared to other secondary plant metabolites [83]. As a result, the nano- and 352 microencapsulation of polyphenols has attracted considerable research attention [84]. 353 Specifically, several successful strategies for stabilizing these compounds through 354 electrospraying have been reported. Table 1 lists the matrices and conditions used to encapsulate phenolic compounds within edible electrosprayed encapsulation matrices, together with the obtained encapsulation efficiencies.

357

One of the phenolics that has been encapsulated through electropraying is (-)-epigallocatechin gallate (EGCG), the most bioactive and abundant catechin in green tea leaves [85]. The first electrosprayed matrix used for this purpose was a protein, namely gelatin [3]. Proteins, and 361 particularly gelatin, can strongly interact with polyphenols [86]. On the other hand, polyphenols are often found as protein-polyphenols complexes within natural foods, and this has been reported to enhance their stability [87]. Accordingly, electrosprayed proteins may be ideal encapsulation vehicles for polyphenols. Moreover, gelatin formulations must be acidified to be processed by electrospraying (cf. Section 3.1), which also has a positive impact in the stability of EGCG [88]. The results showed that EGCG could be efficiency encapsulated within the obtained electrosprayed gelatin submicroparticles (encapsulation efficiency was nearly $100 \%$ ), which was partly attributed to the good solubility of the polyphenol in the gelatin formulation. Moreover, it was seen that this encapsulation strategy effectively enhanced the stability of the catechin when exposed to adverse conditions [3]. A diffusion mechanism was predominant in the release of polyphenols from the electrosprayed capsules in three different release media [3]. 
374 Other wall materials which have been explored to encapsulate EGCG by electrospraying

375 include chitosan [37] or zein [89], and both the uniaxial [3] and coaxial [50] electrospraying 376 configurations have been exploited to this end. Being EGCG a water-soluble bioactive compound, all the proposed systems yielded considerably high encapsulation efficiencies (> 76\%). Furthermore, electrospraying proved to be an effective encapsulation approach to enhance the stability of the polyphenol in aqueous media and upon simulated in-vitro digestion [50].

The encapsulation of complex green tea extracts rather than a single purified catechin by electrospraying has also been addressed, using gelatin and zein as wall materials too. Electrosprayed zein nanocapsules were reported to significantly enhance the stability of the green tea catechins during in-vitro digestion as well as their bioavailability assessed in Caco-2 cell models [48]. In order to study the real impact of microencapsulation through electrospraying on the stabilization of green tea catechins in real food processing conditions, Gómez-Mascaraque, et al. (2017) incorporated gelatin and zein electrosprayed capsules containing a green tea extract into a biscuit dough which was then subjected to a real baking process. Although the protective effect observed in the in-vitro tests (thermal treatment of the particles in the absence of food matrix) could not be confirmed in the real baking process within the dough due to the complexity of the system under study, the results showed that the addition of electrosprayed protein capsules did not have a negative impact on the consumers' acceptability of the biscuits [64], which is also an important requirement for the intended use of the encapsulation structures in food products.

397 Electrosprayed zein particles were also used to encapsulate a phenolics-rich extract obtained 398 from Geoffroea decorticans (chañar), which was proposed as a potential ingredient for 
functional foods development [49]. Once more, the results showed that the electrospraying process did not negatively affect the bioactivity of the extract and that it was an effective encapsulation approach to protect the phenolic compounds during simulated digestion invitro. A black carrot extract, rich in anthocyanins, was also encapsulated through electrospraying. In this case, gelatin/chitosan blends were selected as wall materials, showing that the release profile of the anthocyanins could be modulated by adjusting the protein/polysaccharide ratio [76].

Curcuminoids are another type of phenolic compounds which involve an additional challenge as compared to the previous flavonoids or phenolic acids, since they are hydrophobic and, thus, insoluble in aqueous media. As a result, zein was initially proposed as wall matrix to encapsulate curcumin through electrospraying, as this ingredient is soluble in ethanol. Encapsulation efficiencies of up to $90 \%$ could be achieved using this strategy, and the developed zein capsules proved to successfully enhance the dispersability of curcumin in milk [51]. Curcumin could also be incorporated within electrosprayed gelatin capsules by using a mixture of solvents (water/acetic acid/ethanol) in which both the wall material and the phenolic compound were soluble. This strategy, which reportedly improved the antimicrobial and antioxidant activities of curcumin [90], also enhanced its dispersability in a fish-derived gelled food product [91].

In order to facilitate encapsulation of curcumin within electrosprayed biopolymers which can only be processed from aqueous solutions, Gómez-Mascaraque, et al. (2017) developed a dual encapsulation strategy in which the lipophilic ingredient was first incorporated within liposome vesicles, which were then further encapsulated by electrospraying within a WPC matrix. This approach did not only improve the bioaccessibility of curcumin in-vitro, but also its stability in 
424 aqueous solution [58]. Subsequent efforts were made towards the design of a system for the 425 continuous production of these hybrid encapsulation vehicles, by combining the 426 electrospraying and microfluidic technologies [59]. 
Table 1. Plant-derived phenolic compounds encapsulated within electrosprayed capsules

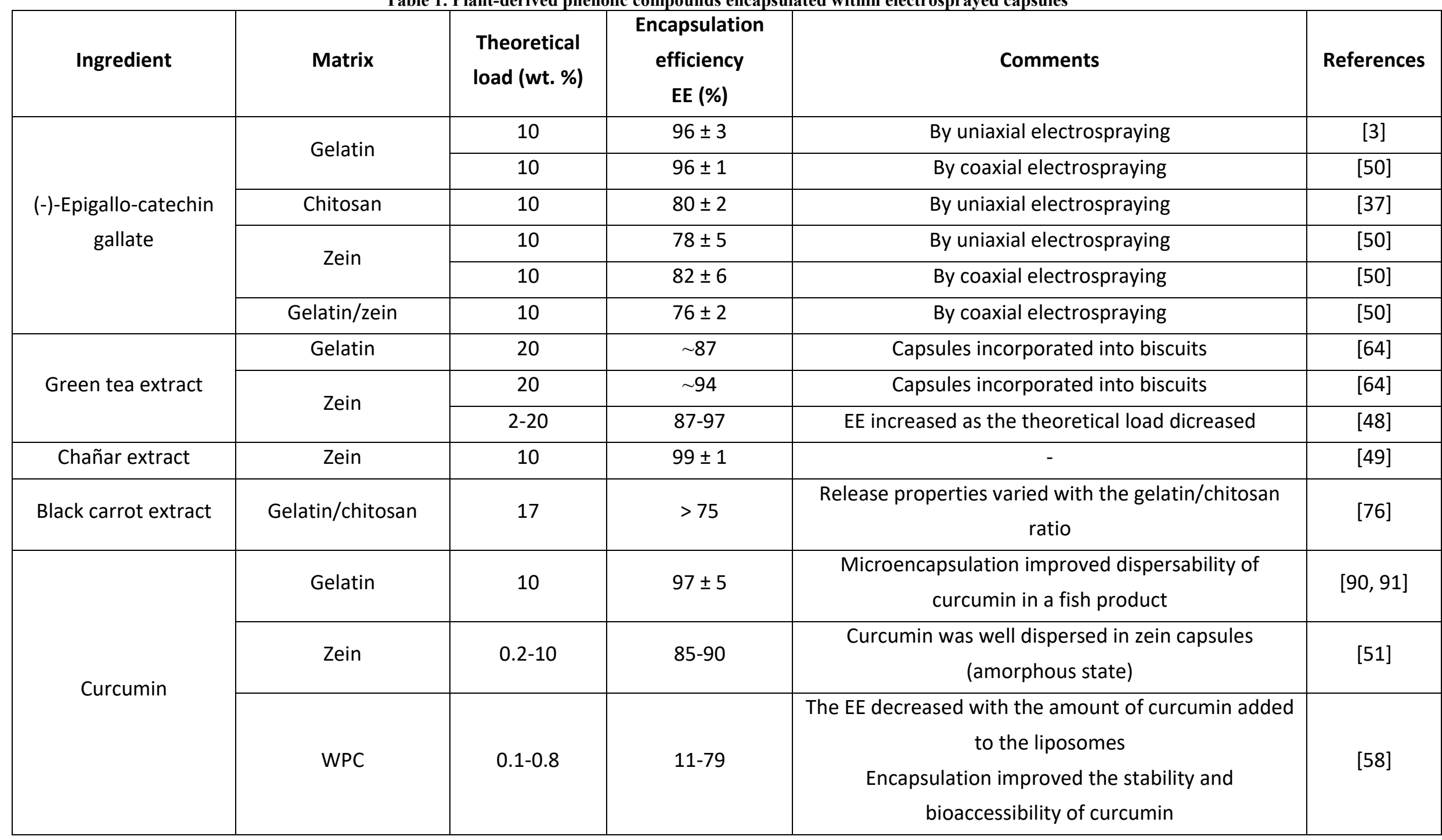




\subsection{Encapsulation of $\omega-3$ fatty acids from vegetable origin}

$\omega-3$ fatty acids are essential nutrients with many attributed health benefits such as prevention and treatment of arthritis, inflammatory and autoimmune diseases, heart disease or cancer [92]. Although many of the $\omega-3$ fatty acids we consume have a non-vegetable origin, mainly from fish oils, plants are also a rich source of bioactive $\omega-3$ fatty acids, being the main one $\alpha$ linolenic acid $[93,94]$. Table 2 summarizes the matrices used to encapsulate $\alpha$-linolenic acid and other interesting fatty acids and oils within edible electrosprayed encapsulation matrices, together with the obtained encapsulation efficiencies.

Similarly to curcumin, $\alpha$-linolenic acid is also soluble in ethanol, so one of the strategies used to encapsulate it through electrospraying consisted of using zein as wall material [50]. In this case, both the uniaxial and coaxial approaches were explored in order to compare them. Moreover, two different systems were produced by coaxial electrospraying, both of them based on a zein core containing the fatty acid, but differing on the protein used as shell material, either zein or gelatin. Although all the proposed systems were effective in stabilizing $\alpha$-linolenic against thermal degradation, coaxial electrospraying yielded better encapsulation efficiencies, and the hybrid zein/gelatin system exhibited the best protective effect [50].

Emulsion-electrospraying has also been exploited for the encapsulation of $\alpha$-linolenic acid using different proteins as wall materials (WPC, SPI and gelatin), and the results were compared to those obtained using spray-drying [56]. Due to the thermosensitivity of the fatty acid, it was completely degraded upon spray-drying, while electrospraying was shown to be a suitable alternative yielding encapsulation efficiencies of up to $67 \pm 5 \%$. Moreover, the electrosprayed protein capsules (except those made from gelatin, which had to be processed 
in acidic conditions) were effective in retarding the thermal oxidation of the bioactive ingredient. 
Table 2. Various plant-derived food ingredients encapsulated within electrosprayed capsules

\begin{tabular}{|c|c|c|c|c|c|}
\hline Ingredient & Matrix & $\begin{array}{l}\text { Theoretical } \\
\text { load (wt. \%) }\end{array}$ & $\begin{array}{c}\text { Encapsulation } \\
\text { efficiency } \\
\text { EE (\%) }\end{array}$ & Comments & References \\
\hline \multirow{6}{*}{$\alpha$-linolenic acid } & \multirow{2}{*}{ Zein } & \multirow{2}{*}{10} & $67 \pm 2$ & By uniaxial electrospraying & \multirow{3}{*}[50]{} \\
\hline & & & $89 \pm 10$ & \multirow{2}{*}{ By coaxial electrospraying } & \\
\hline & Gelatin/zein & 10 & $75 \pm 4$ & & \\
\hline & WPC & 10 & $50-67$ & & \multirow{3}{*}[56]{} \\
\hline & Soy protein isolate & 10 & $30-61$ & The 5 yorind denending & \\
\hline & Gelatin & 10 & $23-40$ & 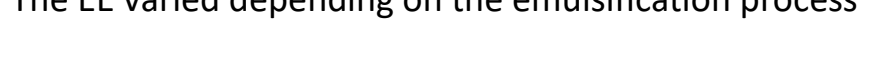 & \\
\hline \multirow{2}{*}{ Folic acid } & WPC & 1.5 & $81 \pm 13$ & \multirow{2}{*}{$\begin{array}{l}\text { WPC yielded higher EE and provided greater } \\
\text { protection than starch }\end{array}$} & \multirow{2}{*}[21]{} \\
\hline & Resistant starch & 1.5 & $44 \pm 6$ & & \\
\hline D-limonene & $\begin{array}{c}\text { Alyssum } \\
\text { homolocarpum seed } \\
\text { gum }\end{array}$ & $9-17$ & $73-93$ & Reported good storage stability of the ingredient & {$[69,95]$} \\
\hline
\end{tabular}

* First value refers to total ingredient in the electrosprayed capsules. Value in brackets refers only to ingredient in the cores, disregarding the amount located on the surface 


\subsection{Encapsulation of carotenoids}

Carotenoids are natural pigments which may help preventing certain diseases, particularly certain types of cancer and eye diseases, when consumed in sufficient levels [96, 97]. Table 3 lists the encapsulation matrices used to encapsulate carotenoids within edible electrosprayed encapsulation matrices, and the obtained encapsulation efficiencies.

The first reported carotenoid incorporated within electrosprayed edible capsules was $\beta$ carotene, which was encapsulated using WPC as protective matrix [39]. Since $\beta$-carotene is a lipophilic compound and, thus, it is not soluble in the aqueous whey protein dispersions, the authors developed a strategy for its incorporation which consisted in dissolving it in glycerol prior to its incorporation within the protein formulation. Although the theoretical load of the capsules was very low (0.1 wt.\%) and only an indirect method was used to assess the encapsulation efficiency, this was the first proof of concept of the feasibility of incorporating bioactive carotenoids in edible electrosprayed matrices.

Soon after, Pérez-Masiá, et al. (2015) encapsulated lycopene within WPC, dextran and chitosan matrices by electrospraying, and also by spray-drying for comparison purposes. They found that, due to the thermosensitiveness of lycopene, lower encapsulation efficiencies were obtained through spray-drying [55]. These results emphasized the convenience of electrospraying for the microencapsulation of thermosensitive ingredients, as this electrohydrodynamic process is normally carried out under mild temperature conditions. It was also found that the greatest encapsulation efficiency and protective effect was obtained using the protein as wall material, which was attributed to its ability to interact with the bioactive ingredient. 
In another work, Gómez-Mascaraque, et al. (2017) used two different proteins, WPC and zein, to assess the potential of the emulsion-electrospraying encapsulation technique to improve the bioaccesibility of $\beta$-carotene [47], which is very low in its free form [4]. Indeed, the bioaccessibility of the carotenoid could be enhanced, and the results showed that the gravitational stability of the emulsions prepared to disperse the lipophilic compound in the protein formulations dramatically impacted the encapsulation efficiency of the systems. These results emphasized the need of thoroughly optimizing the feed formulations to be electrosprayed [47]. 
Table 3. Carotenoids encapsulated within electrosprayed capsules

\begin{tabular}{|c|c|c|c|c|c|}
\hline Ingredient & Matrix & $\begin{array}{l}\text { Theoretical } \\
\text { load (wt. \%) }\end{array}$ & $\begin{array}{c}\text { Encapsulation } \\
\text { efficiency } \\
\text { EE (\%) }\end{array}$ & Comments & References \\
\hline \multirow{3}{*}{$\beta$-carotene } & \multirow{2}{*}{$\begin{array}{c}\text { Whey protein } \\
\text { concentrate (WPC) }\end{array}$} & 0.1 & $\begin{array}{c}90 \% \text { (indirect } \\
\text { measurement of } \\
\text { glycerol) }\end{array}$ & Ingredient dissolved in glycerol & [39] \\
\hline & & 2.5 & $57 \pm 1(22 \pm 3)^{*}$ & $\begin{array}{l}\text { Ingredient dissolved in soy bean oil and encapsulated } \\
\text { by emulsion-electrospraying }\end{array}$ & [47] \\
\hline & Zein & 2.5 & $74 \pm 3(33 \pm 9)^{*}$ & $\begin{array}{l}\text { Ingredient dissolved in soy bean oil and encapsulated } \\
\text { by emulsion-electrospraying }\end{array}$ & [47] \\
\hline \multirow{5}{*}{ Lycopene } & \multirow{2}{*}{ WPC } & 0.4 & $72 \pm 7$ & $\begin{array}{l}\text { Ingredient dissolved in soy bean oil and encapsulated } \\
\text { by emulsion-electrospraying }\end{array}$ & \multirow{5}{*}[55]{} \\
\hline & & 0.3 & $75 \pm 33$ & $\begin{array}{l}\text { Ingredient dissolved in soy bean oil and encapsulated } \\
\text { by coaxial electrospraying }\end{array}$ & \\
\hline & \multirow{2}{*}{ Dextran } & 0.5 & $26 \pm 9$ & $\begin{array}{l}\text { Ingredient dissolved in soy bean oil and encapsulated } \\
\text { by emulsion-electrospraying }\end{array}$ & \\
\hline & & 0.4 & $58 \pm 18$ & $\begin{array}{l}\text { Ingredient dissolved in soy bean oil and encapsulated } \\
\text { by coaxial electrospraying }\end{array}$ & \\
\hline & Chitosan & 1.5 & Negligible & Ingredient degraded due to highly acidic conditions & \\
\hline
\end{tabular}

* First value refers to total ingredient in the electrosprayed capsules. Value in brackets refers only to ingredient in the cores, disregarding the amount located on the surface 


\subsection{Encapsulation of other plant-derived ingredients}

Plant-derived vitamins have also been the subject of microencapsulation through electrospraying. This is the case of folic acid. Pérez-Masiá, et al. (2015) encapsulated folic acid within WPC and resistant starch using both the electrospraying and spray-drying techniques, in order to compare their performance [21]. They found that similar encapsulation efficiencies (cf. Table 2) were obtained through both technologies, which were higher when the protein was used as wall material. This was attributed to its ability to interact with the vitamin. Moreover, WPC was more effective than the polysaccharide in stabilizing folic acid against degradation during storage.

D-limonene, which is a flavouring compound found in citrus, has been suggested to have functional properties, such as positive effects in the prevention and treatment of some types of cancer [98]. Khoshakhlagh, et al. (2018) encapsulated D-limonene through emulsionelectrospraying using Alyssum homolocarpum seed gum as wall material. Despite being a volatile compound and even though the concentration of wall material in the emulsions was quite low (0.5 wt.\%), the authors reported a high encapsulation efficiency (cf. Table 2) and good storage stability ( $>90 \%$ of the compound was retained after 90 days of storage at $4{ }^{\circ} \mathrm{C}$, and $>80 \%$ at $25^{\circ} \mathrm{C}$ ) [69]. The authors also found that the encapsulation efficiency decreased with increasing D-limonene loadings [95], as generally occurs when the ingredient/wall material ratio increases in encapsulation systems.

\section{Future trends and concluding remarks}

Electrospraying is a drying encapsulation technique with a great potential in the field of nutraceuticals and functional foods. It represents an attractive alternative to other existing technologies, especially for thermosensitive bioactive ingredients whose activity can be 
affected by the thermal treatment to which they are subjected upon processing through the more common spray-drying technique. However, electrospraying also involves a series of challenges, mainly related to the need of thoroughly optimizing the processing conditions and feed formulations for each particular wall material/bioactive ingredient system.

In the last decade, intensive scientific efforts have been made to develop a wide range of electrosprayed microstructures using different proteins and polysaccharides as wall materials, yielding promising results in line with their potential application as nano- and microencapsulation vehicles for plant-derived functional ingredients through electrospraying. Nonetheless, there is still an opportunity to widen the list of electrosprayed edible biopolymers, which is still limited. New approaches and strategies are expected to be proposed in order to enhance the electrosprayability of alternative wall materials. Additionally, future works are expected to focus on the development of electrosprayed encapsulation structures based on biopolymer blends, since this approach has demonstrated to be a good strategy to modulate the performance of the obtained materials as delivery vehicles, and only limited published works have focused on this topic to date.

On the other hand, most of the research works dealing with the nano- or microencapsulation of plant-derived bioactive ingredients for the development of functional foods and nutraceuticals assessed the behaviour of the encapsulation structures in-vitro, using simple food simulants instead of complex food matrices. Evaluating their performance in real food systems is the necessary next step, in order to confirm the suitability of the proposed delivery systems to achieve the desired goals. 
Finally, as the application of electrospraying for microencapsulation is still in its exploratory phase, most of the research works in this field have been conducted at lab-scale, using one single capillary nozzle. Nonetheless, due to the increased interest in the electrohydrodynamic processing techniques for production of different materials in various sectors, many technological solutions are already being proposed to scale up the production [99]. These industrial solutions have not been yet applied for the production of electrosprayed encapsulation structures in the food sector, and this challenge should be also addressed in the future.

\section{References}

[1] Rhodes, M.; Price, K., Phytochemicals: classification and occurrence. Encyclopedia of Human Nutrition (Sadler, MJ, Strain, JJ \& Caballero, B., eds.), 1999, 1539-1549.

[2] Ozen, A.E.; Pons, A.; Tur, J.A., Worldwide consumption of functional foods: A systematic review. Nutrition Reviews, 2012, 70, (8), 472-481.

[3] Gómez-Mascaraque, L.G.; Lagarón, J.M.; López-Rubio, A., Electrosprayed gelatin submicroparticles as edible carriers for the encapsulation of polyphenols of interest in functional foods. Food Hydrocolloids, 2015, 49, (Supplement C), 42-52.

[4] Deng, X.X.; Chen, Z.; Huang, Q.; Fu, X.; Tang, C.H., Spray-drying microencapsulation of $\beta$-carotene by soy protein isolate and/or OSA-modified starch. Journal of Applied Polymer Science, 2014, 131, (12).

[5] Gómez-Mascaraque, L.G.; López-Rubio, A., Protein-based emulsion electrosprayed micro- and submicroparticles for the encapsulation and stabilization of thermosensitive hydrophobic bioactives. Journal of Colloid and Interface Science, 2016, 465, 259-270.

[6] Lafarga, T.; Hayes, M., Bioactive protein hydrolysates in the functional food ingredient industry: Overcoming current challenges. Food Reviews International, 2017, 33, (3), 217-246.

[7] Jiménez-Martín, E.; Gharsallaoui, A.; Pérez-Palacios, T.; Carrascal, J.; Rojas, T., Suitability of using monolayered and multilayered emulsions for microencapsulation of $\omega-3$ fatty acids by spray drying: Effect of storage at different temperatures. Food and Bioprocess Technology, 2014, 8, (1), 100-111.

[8] Jafari, S.M. Nanoencapsulation Technologies for the Food and Nutraceutical Industries. Academic Press, 2017.

[9] Ye, A.; Cui, J.; Taneja, A.; Zhu, X.; Singh, H., Evaluation of processed cheese fortified with fish oil emulsion. Food Research International, 2009, 42, (8), 1093-1098.

[10] Jafari, S.M. In Nanoencapsulation Technologies for the Food and Nutraceutical Industries. Jafari, S.M., Ed.; Academic Press, 2017, pp 1-34.

[11] Đorđević, V.; Balanč, B.; Belščak-Cvitanović, A.; Lević, S.; Trifković, K.; Kalušević, A.; Kostić, I.; Komes, D.; Bugarski, B.; Nedović, V., Trends in encapsulation technologies for delivery of food bioactive compounds. Food Engineering Reviews, 2015, 7, (4), 452-490. 
[12] Gharsallaoui, A.; Roudaut, G.; Chambin, O.; Voilley, A.; Saurel, R., Applications of spraydrying in microencapsulation of food ingredients: An overview. Food Research International, 2007, 40, (9), 1107-1121.

[13] Nedović, V.; Kalušević, A.; Manojlović, V.; Petrović, T.; Bugarski, B. In Advances in food process engineering research and applications; Springer, 2013, pp 229-253.

[14] Zuidam, N.J.; Shimoni, E. In Encapsulation technologies for active food ingredients and food processing; Springer, 2010, pp 3-29.

[15] Kwak, H.-S., Overview of nano-and microencapsulation for foods. Nano-and Microencapsulation for Foods, 2014, 1-14.

[16] Silva, D.; FAVARO-TRINDADE, C.; Rocha, G.; Thomazini, M., Microencapsulation of lycopene by gelatin-pectin complex coacervation. Journal of Food Processing and Preservation, 2012, 36, (2), 185-190.

[17] Leclercq, S.; Harlander, K.R.; Reineccius, G.A., Formation and characterization of microcapsules by complex coacervation with liquid or solid aroma cores. Flavour and Fragrance Journal, 2009, 24, (1), 17-24.

[18] Pérez-Masiá, R.; Lagaron, J.; Lopez-Rubio, A., Morphology and Stability of Edible Lycopene-Containing Micro- and Nanocapsules Produced Through Electrospraying and Spray Drying. Food and Bioprocess Technology, 2015, 8, (2), 459-470.

[19] López-Rubio, A.; Lagaron, J.M., Whey protein capsules obtained through electrospraying for the encapsulation of bioactives. Innovative Food Science \& Emerging Technologies, 2012, 13, (0), 200-206.

[20] Bock, N.; Dargaville, T.R.; Woodruff, M.A., Electrospraying of polymers with therapeutic molecules: State of the art. Progress in Polymer Science, 2012, 37, (11), 1510-1551.

[21] Pérez-Masiá, R.; López-Nicolás, R.; Periago, M.J.; Ros, G.; Lagaron, J.M.; López-Rubio, A., Encapsulation of folic acid in food hydrocolloids through nanospray drying and electrospraying for nutraceutical applications. Food Chemistry, 2015, 168, 124-133.

[22] Tapia-Hernández, J.A.; Torres-Chávez, P.I.; Ramírez-Wong, B.; Rascón-Chu, A.; Plascencia-Jatomea, M.; Barreras-Urbina, C.G.; Rangel-Vázquez, N.A.; Rodríguez-Félix, F., Micro-and nanoparticles by electrospray: advances and applications in foods. Journal of agricultural and food chemistry, 2015, 63, (19), 4699-4707.

[23] Gómez-Mascaraque, L.G., J. Ambrosio-Martín, M. J. Fabra, R. Pérez-Masiá, J. M. Lagaron and A. López-Rubio. In Nanotechnology in Nutraceuticals; CRC Press, 2016, pp 373395.

[24] Chakraborty, S.; Liao, I.C.; Adler, A.; Leong, K.W., Electrohydrodynamics: A facile technique to fabricate drug delivery systems. Advanced Drug Delivery Reviews, 2009, 61, (12), 1043-1054.

[25] Jaworek, A.; Sobczyk, A.T., Electrospraying route to nanotechnology: An overview. Journal of Electrostatics, 2008, 66, (3-4), 197-219.

[26] Bhushani, J.A.; Anandharamakrishnan, C., Electrospinning and electrospraying techniques: Potential food based applications. Trends in food science \& technology, 2014, 38, (1), 21-33.

[27] Rosell-Llompart, J.; Grifoll, J.; Loscertales, I.G., Electrosprays in the cone-jet mode: from Taylor cone formation to spray development. Journal of Aerosol Science, 2018.

[28] Bhardwaj, N.; Kundu, S.C., Electrospinning: A fascinating fiber fabrication technique. Biotechnology Advances, 2010, 28, (3), 325-347.

[29] Tapia-Hernández, J.A.; Rodríguez-Félix, F.; Katouzian, I. In Nanoencapsulation Technologies for the Food and Nutraceutical Industries. Jafari, S.M., Ed.; Academic Press, 2017, pp 320-345. 
[30] Bhushani, J.A.; Anandharamakrishnan, C., Electrospinning and electrospraying techniques: Potential food based applications. Trends in Food Science \& Technology 2014, 113.

[31] Alehosseini, A.; Ghorani, B.; Sarabi-Jamab, M.; Tucker, N., Principles of electrospraying: A new approach in protection of bioactive compounds in foods. Critical Reviews in Food Science and Nutrition, 2017, 1-18.

[32] Kriegel, C.; Arrechi, A.; Kit, K.; McClements, D.; Weiss, J., Fabrication, functionalization, and application of electrospun biopolymer nanofibers. Critical reviews in food science and nutrition, 2008, 48, (8), 775-797.

[33] Dumitriu, R.P.; Mitchell, G.R.; Davis, F.J.; Vasile, C., Functionalized Coatings by Electrospinning for Anti-oxidant Food Packaging. Procedia Manufacturing, 2017, 12, 59-65.

[34] Fabra, M.J.; López-Rubio, A.; Lagaron, J.M., Use of the electrohydrodynamic process to develop active/bioactive bilayer films for food packaging applications. Food Hydrocolloids, 2016, 55, 11-18.

[35] Zaeim, D.; Sarabi-Jamab, M.; Ghorani, B.; Kadkhodaee, R.; Tromp, R.H., Electrospray assisted fabrication of hydrogel microcapsules by single-and double-stage procedures for encapsulation of probiotics. Food and bioproducts processing, 2017, 102, 250-259.

[36] Doshi, J.; Reneker, D.H., Electrospinning process and applications of electrospun fibers. Journal of electrostatics, 1995, 35, (2-3), 151-160.

[37] Gómez-Mascaraque, L.G.; Sanchez, G.; López-Rubio, A., Impact of molecular weight on the formation of electrosprayed chitosan microcapsules as delivery vehicles for bioactive compounds. Carbohydrate Polymers, 2016, 150, (Supplement C), 121-130.

[38] Ramakrishna, S. An introduction to electrospinning and nanofibers. World Scientific, 2005.

[39] López-Rubio, A.; Lagaron, J.M., Whey protein capsules obtained through electrospraying for the encapsulation of bioactives. Innovative food science \& emerging technologies, 2012, 13, 200-206.

[40] Pérez-Masiá, R.; Lagaron, J.M.; López-Rubio, A., Surfactant-aided electrospraying of low molecular weight carbohydrate polymers from aqueous solutions. Carbohydrate polymers, 2014, 101, 249-255.

[41] Pérez-Masiá, R.; Lagaron, J.; López-Rubio, A., Development and Optimization of Novel Encapsulation Structures of Interest in Functional Foods Through Electrospraying. Food and Bioprocess Technology, 2014, 7, (11), 3236-3245.

[42] Ma, J.-J.; Mao, X.-Y.; Wang, Q.; Yang, S.; Zhang, D.; Chen, S.-W.; Li, Y.-H., Effect of spray drying and freeze drying on the immunomodulatory activity, bitter taste and hygroscopicity of hydrolysate derived from whey protein concentrate. LWT-Food Science and Technology, 2014, 56, (2), 296-302.

[43] Malaki Nik, A.; Wright, A.J.; Corredig, M., Interfacial design of protein-stabilized emulsions for optimal delivery of nutrients. Food \& Function, 2010, 1, (2), 141-148.

[44] Livney, Y.D., Milk proteins as vehicles for bioactives. Current Opinion in Colloid \& Interface Science, 2010, 15, (1), 73-83.

[45] McClements, D.J., Protein-stabilized emulsions. Current Opinion in Colloid \& Interface Science, 2004, 9, (5), 305-313.

[46] Torres-Giner, S.; Martinez-Abad, A.; Ocio, M.J.; Lagaron, J.M., Stabilization of a nutraceutical omega-3 fatty acid by encapsulation in ultrathin electrosprayed zein prolamine. Journal of food science, 2010, 75, (6).

[47] Gómez-Mascaraque, L.G.; Perez-Masiá, R.; González-Barrio, R.; Periago, M.J.; LópezRubio, A., Potential of microencapsulation through emulsion-electrospraying to improve the bioaccesibility of $\beta$-carotene. Food Hydrocolloids, 2017, 73, 1-12. 
[48] Bhushani, J.A.; Kurrey, N.K.; Anandharamakrishnan, C., Nanoencapsulation of green tea catechins by electrospraying technique and its effect on controlled release and in-vitro permeability. Journal of Food Engineering, 2017, 199, 82-92.

[49] Costamagna, M.S.; Gómez-Mascaraque, L.G.; Zampini, I.C.; Alberto, M.R.; Pérez, J.; López-Rubio, A.; Isla, M.I., Microencapsulated chañar phenolics: A potential ingredient for functional foods development. Journal of Functional Foods, 2017, 37, (Supplement C), 523-530. [50] Gómez-Mascaraque, L.G.; Tordera, F.; Fabra, M.J.; Martínez-Sanz, M.; Lopez-Rubio, A., Coaxial electrospraying of biopolymers as a strategy to improve protection of bioactive food ingredients. Innovative Food Science \& Emerging Technologies, 2018.

[51] Gomez-Estaca, J.; Balaguer, M.; Gavara, R.; Hernandez-Munoz, P., Formation of zein nanoparticles by electrohydrodynamic atomization: Effect of the main processing variables and suitability for encapsulating the food coloring and active ingredient curcumin. Food Hydrocolloids, 2012, 28, (1), 82-91.

[52] Liu, Z.-P.; Zhang, Y.-Y.; Yu, D.-G.; Wu, D.; Li, H.-L., Fabrication of sustained-release zein nanoparticles via modified coaxial electrospraying. Chemical Engineering Journal, 2018, 334, 807-816.

[53] Zhang, L.; Huang, J.; Si, T.; Xu, R.X., Coaxial electrospray of microparticles and nanoparticles for biomedical applications. Expert review of medical devices, 2012, 9, (6), 595612.

[54] Zhang, C.; Yao, Z.-C.; Ding, Q.; Choi, J.J.; Ahmad, Z.; Chang, M.-W.; Li, J.-S., Tri-Needle Coaxial Electrospray Engineering of Magnetic Polymer Yolk-Shell Particles Possessing DualImaging Modality, Multiagent Compartments, and Trigger Release Potential. ACS applied materials \& interfaces, 2017, 9, (25), 21485-21495.

[55] Pérez-Masiá, R.; Lagaron, J.M.; Lopez-Rubio, A., Morphology and stability of edible lycopene-containing micro-and nanocapsules produced through electrospraying and spray drying. Food and bioprocess technology, 2015, 8, (2), 459-470.

[56] Gómez-Mascaraque, L.G.; López-Rubio, A., Protein-based emulsion electrosprayed micro- and submicroparticles for the encapsulation and stabilization of thermosensitive hydrophobic bioactives. Journal of Colloid and Interface Science, 2016, 465, (Supplement C), 259-270.

[57] López-Rubio, A.; Sanchez, E.; Wilkanowicz, S.; Sanz, Y.; Lagaron, J.M., Electrospinning as a useful technique for the encapsulation of living bifidobacteria in food hydrocolloids. Food Hydrocolloids, 2012, 28, (1), 159-167.

[58] Gómez-Mascaraque, L.G.; Sipoli, C.C.; de La Torre, L.G.; López-Rubio, A., Microencapsulation structures based on protein-coated liposomes obtained through electrospraying for the stabilization and improved bioaccessibility of curcumin. Food chemistry, 2017, 233, 343-350.

[59] Gómez-Mascaraque, L.G.; Sipoli, C.C.; de La Torre, L.G.; López-Rubio, A., A step forward towards the design of a continuous process to produce hybrid liposome/protein microcapsules. Journal of Food Engineering, 2017, 214, 175-181.

[60] Gómez-Mascaraque, L.G.; Llavata-Cabrero, B.; Martínez-Sanz, M.; Fabra, M.J.; LópezRubio, A., Self-assembled gelatin-t-carrageenan encapsulation structures for intestinaltargeted release applications. Journal of Colloid and Interface Science, 2018, 517, 113-123.

[61] Erencia, M.; Cano, F.; Tornero, J.A.; Macanás, J.; Carrillo, F., Resolving the electrospinnability zones and diameter prediction for the electrospinning of the gelatin/water/acetic acid system. Langmuir, 2014, 30, (24), 7198-7205.

[62] Okutan, N.; Terzi, P.; Altay, F., Affecting parameters on electrospinning process and characterization of electrospun gelatin nanofibers. Food Hydrocolloids, 2014, 39, 19-26. 
[63] Gómez-Mascaraque, L.G.; Ambrosio-Martín, J.; Perez-Masiá, R.; Lopez-Rubio, A., Impact of Acetic Acid on the Survival of L. plantarum upon Microencapsulation by Coaxial Electrospraying. Journal of healthcare engineering, 2017, 2017.

[64] Gómez-Mascaraque, L.G.; Hernández-Rojas, M.; Tarancón, P.; Tenon, M.; Feuillère, N.; Ruiz, J.F.V.; Fiszman, S.; López-Rubio, A., Impact of microencapsulation within electrosprayed proteins on the formulation of green tea extract-enriched biscuits. LWT-Food Science and Technology, 2017, 81, 77-86.

[65] Gómez-Mascaraque, L.G.; Fabra, M.J.; Castro-Mayorga, J.L.; Sánchez, G.; MartínezSanz, M.; López-Rubio, A. In Biopolymers for Food Design; Elsevier, 2018, pp 33-64.

[66] Fathi, M.; Martin, A.; McClements, D.J., Nanoencapsulation of food ingredients using carbohydrate based delivery systems. Trends in Food Science \& Technology, 2014, 39, (1), 1839.

[67] Mohan, A.; Rajendran, S.R.; He, Q.S.; Bazinet, L.; Udenigwe, C.C., Encapsulation of food protein hydrolysates and peptides: a review. Rsc Advances, 2015, 5, (97), 79270-79278.

[68] Stijnman, A.C.; Bodnar, I.; Tromp, R.H., Electrospinning of food-grade polysaccharides. Food Hydrocolloids, 2011, 25, (5), 1393-1398.

[69] Khoshakhlagh, K.; Mohebbi, M.; Koocheki, A.; Allafchian, A., Encapsulation of Dlimonene in Alyssum homolocarpum seed gum nanocapsules by emulsion electrospraying: Morphology characterization and stability assessment. Bioactive Carbohydrates and Dietary Fibre, 2018.

[70] Zaeim, D.; Sarabi-Jamab, M.; Ghorani, B.; Kadkhodaee, R.; Tromp, R.H., Electrosprayassisted drying of live probiotics in acacia gum microparticles matrix. Carbohydrate Polymers, 2018, 183, 183-191.

[71] Sun, K.; Li, Z., Preparations, properties and applications of chitosan based nanofibers fabricated by electrospinning. Express Polymer Letters, 2011, 5, (4).

[72] Gómez-Mascaraque, L.G.; Méndez, J.A.; Fernández-Gutiérrez, M.; Vázquez, B.; San Román, J., Oxidized dextrins as alternative crosslinking agents for polysaccharides: Application to hydrogels of agarose-chitosan. Acta Biomaterialia, 2014, 10, (2), 798-811.

[73] Moreno, J.A.S.; Mendes, A.C.; Stephansen, K.; Engwer, C.; Goycoolea, F.M.; Boisen, A.; Nielsen, L.H.; Chronakis, I.S., Development of electrosprayed mucoadhesive chitosan microparticles. Carbohydrate polymers, 2018, 190, 240-247.

[74] Zhang, S.; Kawakami, K., One-step preparation of chitosan solid nanoparticles by electrospray deposition. International Journal of Pharmaceutics, 2010, 397, (1), 211-217.

[75] Neha, A.; Syandan, C.; Nikhil, D.; S., K.D., Electrospraying: A facile technique for synthesis of chitosan-based micro/nanospheres for drug delivery applications. Journal of Biomedical Materials Research Part B: Applied Biomaterials, 2009, 88B, (1), 17-31.

[76] Atay, E.; Fabra, M.J.; Martínez-Sanz, M.; Gomez-Mascaraque, L.G.; Altan, A.; LopezRubio, A., Development and characterization of chitosan/gelatin electrosprayed microparticles as food grade delivery vehicles for anthocyanin extracts. Food Hydrocolloids, 2017.

[77] García-Moreno, P.J.; Pelayo, A.; Yu, S.; Busolo, M.; Lagaron, J.M.; Chronakis, I.S.; Jacobsen, C., Physicochemical characterization and oxidative stability of fish oil-loaded electrosprayed capsules: Combined use of whey protein and carbohydrates as wall materials. Journal of Food Engineering, 2018, 231, 42-53.

[78] Maki, K. In Phytochemical Functional Foods. Johnson, I.; Williamson, G., Eds.; Woodhead Publishing, 2003, pp 238-252.

[79] Andersen, M.L.; Lauridsen, R.K.; Skibsted, L.H. In Phytochemical Functional Foods. Johnson, I.; Williamson, G., Eds.; Woodhead Publishing, 2003, pp 315-346.

[80] Xiao, J.; Cao, Y.; Huang, Q., Edible Nanoencapsulation Vehicles for Oral Delivery of Phytochemicals: A Perspective Paper. Journal of Agricultural and Food Chemistry, 2017, 65, (32), 6727-6735. 
[81] Lee, S.J.; Wong, M., Nano-and microencapsulation of phytochemicals. Nano-and Microencapsulation for Foods, 2014, 117-165.

[82] Manach, C.; Scalbert, A.; Morand, C.; Rémésy, C.; Jiménez, L., Polyphenols: food sources and bioavailability. The American Journal of Clinical Nutrition, 2004, 79, (5), 727-747.

[83] Crowe, K.M., Designing functional foods with bioactive polyphenols: Highlighting lessons learned from original plant matrices. Journal of Human Nutrition and Food Science, 2013, 1, (3), 1018.

[84] Faridi Esfanjani, A.; Jafari, S.M., Biopolymer nano-particles and natural nano-carriers for nano-encapsulation of phenolic compounds. Colloids and Surfaces B: Biointerfaces, 2016, $146,532-543$.

[85] Barras, A.; Mezzetti, A.; Richard, A.; Lazzaroni, S.; Roux, S.; Melnyk, P.; Betbeder, D.; Monfilliette-Dupont, N., Formulation and characterization of polyphenol-loaded lipid nanocapsules. International Journal of Pharmaceutics, 2009, 379, (2), 270-277.

[86] Peña, C.; de la Caba, K.; Eceiza, A.; Ruseckaite, R.; Mondragon, I., Enhancing water repellence and mechanical properties of gelatin films by tannin addition. Bioresource Technology, 2010, 101, (17), 6836-6842.

[87] Bandyopadhyay, P.; Ghosh, A.K.; Ghosh, C., Recent developments on polyphenolprotein interactions: effects on tea and coffee taste, antioxidant properties and the digestive system. Food \& function, 2012, 3, (6), 592-605.

[88] Gómez-Mascaraque, L.G.; Soler, C.; Lopez-Rubio, A., Stability and bioaccessibility of EGCG within edible micro-hydrogels. Chitosan vs. gelatin, a comparative study. Food Hydrocolloids, 2016, 61, 128-138.

[89] Gómez-Mascaraque, L.G.; Tordera, F.; Fabra, M.J.; Martínez-Sanz, M.; Lopez-Rubio, A., Coaxial electrospraying of biopolymers as a strategy to improve protection of bioactive food ingredients. Innovative Food Science \& Emerging Technologies, 2019, 51, 2-11.

[90] Gómez-Estaca, J.; Balaguer, M.P.; López-Carballo, G.; Gavara, R.; Hernández-Muñoz, P., Improving antioxidant and antimicrobial properties of curcumin by means of encapsulation in gelatin through electrohydrodynamic atomization. Food Hydrocolloids, 2017, 70, 313-320.

[91] Gómez-Estaca, J.; Gavara, R.; Hernández-Muñoz, P., Encapsulation of curcumin in electrosprayed gelatin microspheres enhances its bioaccessibility and widens its uses in food applications. Innovative Food Science \& Emerging Technologies, 2015, 29, 302-307.

[92] Ursin, V.M., Modification of Plant Lipids for Human Health: Development of Functional Land-Based Omega-3 Fatty Acids. The Journal of Nutrition, 2003, 133, (12), 4271-4274.

[93] Nettleton, J., Omega-3 fatty acids: comparison of plant and seafood sources in human nutrition. Journal of the American Dietetic Association, 1991, 91, (3), 331-337.

[94] Sanders, T.A.B., DHA status of vegetarians. Prostaglandins, Leukotrienes and Essential Fatty Acids, 2009, 81, (2), 137-141.

[95] Khoshakhlagh, K.; Koocheki, A.; Mohebbi, M.; Allafchian, A., Development and characterization of electrosprayed Alyssum homolocarpum seed gum nanoparticles for encapsulation of d-limonene. Journal of Colloid and Interface Science, 2017, 490, 562-575.

[96] Giuseppe, M.; Jesús, P.C.M.; Giovina, C.; Elisabetta, T.; Goñi, C.I.; Anette, B.; Fernando, G.-L.; Begoña, O.-A.; Pia, K.; Massimo, V.; Volker, B.; Esther, M.-M.; Diana, B.; Ulrich, S., Carotenoids: Actual knowledge on food sources, intakes, stability and bioavailability and their protective role in humans. Molecular Nutrition \& Food Research, 2009, 53, (S2), S194-S218.

[97] Johnson, E.J., The role of carotenoids in human health. Nutrition in clinical care, 2002, 5, (2), 56-65.

[98] Miller, J.A.; Thompson, P.A.; Hakim, I.A.; Chow, H.-H.S.; Thomson, C.A., d-Limonene: a bioactive food component from citrus and evidence for a potential role in breast cancer prevention and treatment. Oncology Reviews, 2011, 5, (1), 31-42. 
[99] Zhang, C.; Chang, M.-W.; Ahmad, Z.; Hu, W.; Zhao, D.; Li, J.-S., Stable single device multi-pore electrospraying of polymeric microparticles via controlled electrostatic interactions. RSC Advances, 2015, 5, (107), 87919-87923. 